\title{
Development and optimization of a microbial co-culture system for heterologous indigo biosynthesis
}

Tingting Chen ${ }^{1,2}$, Xiaonan Wang ${ }^{2}$, Lei Zhuang ${ }^{2}$, Alan Shao ${ }^{3}$, Yinghua Lu ${ }^{1,4,5}$ and Haoran Zhang ${ }^{2^{*}}$ (1)

\begin{abstract}
Background: Indigo is a color molecule with a long history of being used as a textile dye. The conventional production methods are facing increasing economy, sustainability and environmental challenges. Therefore, developing a green synthesis method converting renewable feedstocks to indigo using engineered microbes is of great research and application interest. However, the efficiency of the indigo microbial biosynthesis is still low and needs to be improved by proper metabolic engineering strategies.
\end{abstract}

Results: In the present study, we adopted several metabolic engineering strategies to establish an efficient microbial biosynthesis system for converting renewable carbon substrates to indigo. First, a microbial co-culture was developed using two individually engineered $E$. coli strains to accommodate the indigo biosynthesis pathway, and the balancing of the overall pathway was achieved by manipulating the ratio of co-culture strains harboring different pathway modules. Through carbon source optimization and application of biosensor-assisted cell selection circuit, the indigo production was improved significantly. In addition, the global transcription machinery engineering (gTME) approach was utilized to establish a high-performance co-culture variant to further enhance the indigo production. Through the step-wise modification of the established system, the indigo bioproduction reached $104.3 \mathrm{mg} / \mathrm{L}$, which was 11.4fold higher than the parental indigo producing strain.

Conclusion: This work combines modular co-culture engineering, biosensing, and gTME for addressing the challenges of the indigo biosynthesis, which has not been explored before. The findings of this study confirm the effectiveness of the developed approach and offer a new perspective for efficient indigo bioproduction. More broadly, this innovative approach has the potential for wider application in future studies of other valuable biochemicals' biosynthesis.

Keywords: Indigo, E. coli, Modular co-culture engineering, Biosensor, Global transcription machinery engineering

\section{Introduction}

Indigo is an aromatic molecule with deep blue color and has been historically used as a natural dye. Most of the indigo used in industry is currently obtained by either plant extraction or chemical synthetic process [1].

*Correspondence: Haoran.Zhang@rutgers.edu

${ }^{2}$ Department of Chemical and Biochemical Engineering, Rutgers, The State University of New Jersey, Piscataway, NJ 08854, USA

Full list of author information is available at the end of the article
However, these methods need to use massive amount of chemicals as extractant, reactant or catalyst, leading to a series of process economic, sustainability, and environmental problems. To address this challenge, the indigo biosynthesis using renewable materials has been explored as a robust alternative.

The indigo biosynthesis pathway is illustrated in Fig. 1. A carbon substrate (e.g. glucose or glycerol) is first converted to amino acid tryptophan through the central metabolism and the tryptophan pathway. Tryptophan original author(s) and the source, provide a link to the Creative Commons licence, and indicate if changes were made. The images or other third party material in this article are included in the article's Creative Commons licence, unless indicated otherwise in a credit line to the material. If material is not included in the article's Creative Commons licence and your intended use is not permitted by statutory regulation or exceeds the permitted use, you will need to obtain permission directly from the copyright holder. To view a copy of this licence, visit http://creativecommons.org/licenses/by/4.0/. The Creative Commons Public Domain Dedication waiver (http://creativeco mmons.org/publicdomain/zero/1.0/) applies to the data made available in this article, unless otherwise stated in a credit line to the data. 


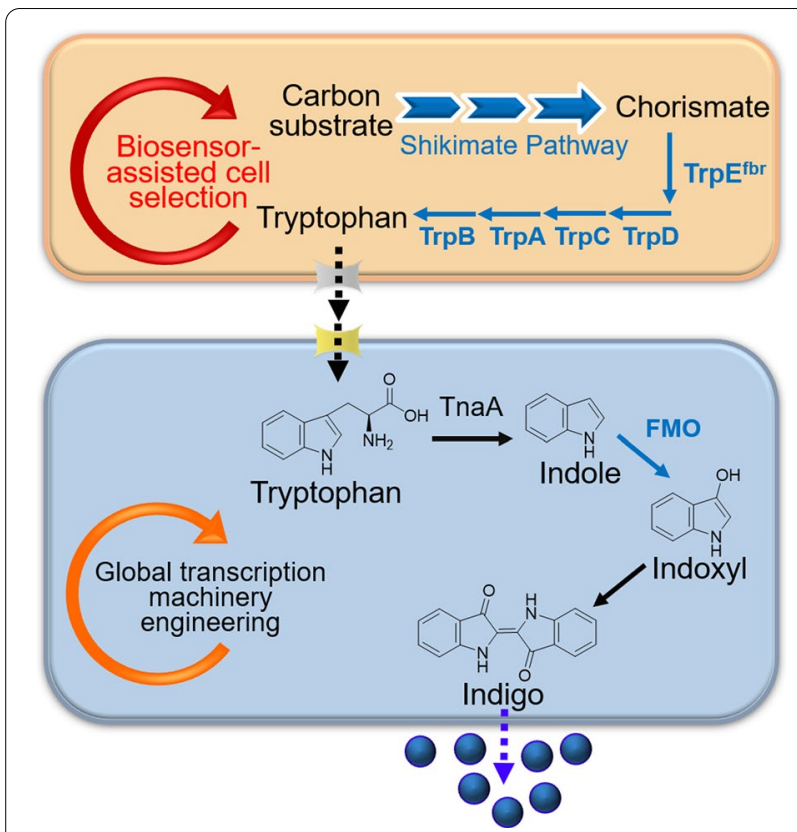

Fig. 1 Design of E. coli-E. coli co-culture system for indigo biosynthesis. The pathway intermediate tryptophan was provided by the upstream strain, and the conversion of tryptophan to indigo was performed by downstream strain. Biosensor-assisted selection circuit was introduced to the upstream strain for enhancing the tryptophan provision. gTME selection was introduced into the downstream strain for improving the indigo production. Blue arrows represent the over-expressed genes

is then further converted to indole by tryptophanase (TnaA) encoded by the tnaA gene. In the presence of a heterologous flavin-containing monooxygenase (FMO), indoxyl and 2-hydroxyindole are produced from indole. Spontaneous dimerization of indoxyl and 2-hydroxyindole results in the formation of indigo and indirubin, respectively [2, 3]. A few microorganisms, including Acinetobacter sp., Comamonas sp., and Rhodococcus opacus B-4, have been utilized to produce indigo in vivo [4-6]. Heterologous biosynthesis of indigo from the precursor indole or tryptophan has also been achieved in E. coli by utilizing phenol hydroxylase [7], naphthalene dioxygenase $[8,9]$, or FMO [10-12]. In addition, several studies have reported the de novo indigo bioproduction using recombinant $E$. coli strains carrying the complete pathway genes leading from glucose to indigo $[8,11,13]$. However, engineering a microbial mono-culture for the indigo biosynthesis often requires extensive genetic and metabolic engineering efforts and is restricted by the metabolic capacity of the recruited microbial hosts to accommodate the need of the biosynthesis pathway.

Recent development of microbial co-culture engineering offers a new perspective and novel opportunities for conducting microbial bioproduction. In particular, the biosynthetic pathway can be divided into separate modules, each of which can be engineered independently. A wealth of co-culture systems has been successfully developed for biosynthesis of various value-added compounds [14-17]. The co-culture engineering approach has several important advantages, such as flexibility for balancing pathway, reduction of metabolic burden, and decrease of undesired interference between pathway modules. Meanwhile, compared with the conventional mono-culturebased approach, co-culture engineering has been proven to be a better platform for adoption of novel engineering strategies. For example, it has been reported that the integration of biosensor-assisted cell selection systems into microbial co-cultures is more effective than into mono-cultures for enhancing the provision of the biosynthesis pathway intermediates and the overall bioproduction performance $[16,18,19]$.

On the other hand, global transcription machinery engineering (gTME) is a powerful engineering approach that tunes the global metabolic network to fit the need of microbial biosynthesis [20]. By engineering transcription factors such as an RNA polymerase subunit, gTME perturbates the global transcriptome of the microbial host, which promotes phenotypic changes of biosynthesis performance. Although there have been many reports using gTME in microbial biosynthesis applications, they focused on engineering the mono-culture of one single microbial host. To our knowledge, there have been no previous efforts utilizing gTME in the context of microbial co-culture. Given the success of co-culture engineering and gTME in the past, their combined use in microbial biosynthesis is an interesting research area that should be explored.

In the present study, the indigo biosynthesis was established in an E. coli-E. coli co-culture, which was subsequently integrated with biosensor-assisted cell selection for improving the biosynthesis. Moreover, gTME approach was utilized to develop a co-culture variant with enhanced biosynthesis capability.

\section{Results}

\section{Developing an E. coli-E. coli co-culture for the indigo} biosynthesis on glucose and glycerol

The indigo biosynthetic pathway consists of tryptophan provision and its conversion to indigo (Fig. 1). To establish an E. coli-E. coli co-culture, the upstream tryptophan provision module and the downstream tryptophan conversion module were introduced into two $E$. coli strains, respectively. A previously constructed tryptophan over-producing strain BTP1 was employed as the upstream co-culture strain [21]. The downstream strain BD was constructed by over-expressing Methylophaga aminisulfidivorans flavin-containing monooxygenase 
(FMO) [11] in a previously engineered $E$. coli strain BH2 [22]. The co-cultivation of these two strains in one culture presented the complete biosynthetic system converting the carbon substrate to the product indigo. Previous studies $[11,23]$ and our own experimental results (Additional file 1: Fig. S1) demonstrated that $30^{\circ} \mathrm{C}$ is the optimal temperature for the indigo synthesis by FMO. Thus, $30^{\circ} \mathrm{C}$ was adopted for all the following experiments.

On the other hand, two renewable carbon substrates, glucose and glycerol, were compared for their effect on the indigo biosynthesis. Glucose is one of the most commonly used sugars for microbial biosynthesis, due to its high abundance and efficient utilization by many microbes. Glycerol is a major byproduct of the biodiesel industry and has been widely used for biosynthesis of a variety of biochemical products in recent years [24-26]. As shown in Fig. 2A, when glucose was employed as the carbon substrate, the BTP1:BD co-culture showed varied indigo production performance at different inoculation ratios. At the optimal ratio of 4:1, $7.7 \mathrm{mg} / \mathrm{L}$ indigo was produced after $48 \mathrm{~h}$ cultivation. For comparison, the FMO gene was over-expressed in BTP1 to generate strain BMC which served as a mono-culture control strain for the de novo bioproduction of indigo. Under the same cultivation conditions, $8.4 \mathrm{mg} / \mathrm{L}$ indigo was produced by BMC.

The indigo production using glycerol as the carbon source is shown in Fig. 2B. At all inoculation ratios, the indigo concentration was higher than when glucose was used. The highest production of $15.9 \mathrm{mg} / \mathrm{L}$ was achieved at the inoculation ratio of 9:1, which was $107 \%$ higher than the optimum production on glucose. The biosynthesis improvement hereby indicated that glycerol is a better carbon source for this co-culture system. In the meantime, the monoculture control (BMC) produced $11.3 \mathrm{mg} / \mathrm{L}$ indigo from $5 \mathrm{~g} / \mathrm{L}$ glycerol, $41 \%$ lower than the production by the BTP1: BD co-culture system.

\section{Utilizing biosensor-assisted cell selection systems to improve the indigo production}

Biosensor-assisted cell selection has been proven to be an effective approach to improve the quality of the microbial population and enhance the microbial biosynthesis performance [16, 18, 21, 27-29]. For this study, this approach was adopted to engineer the upstream co-culture strain. Specifically, the growth of the engineered strain was directed by a growth-regulating gene whose expression was under the control of a tryptophan-responsive biosensor: low tryptophan-producing cells were inhibited for growth, whereas the growth of high tryptophan producing cells were promoted. Two tryptophan-responsive biosensor-assisted cell selection systems, tnaC-tet $A$ and TrpR-Pmtr-hipA, were introduced into the upstream strain (Additional file 1: Fig. S2). For the tnaC-tetA system, the leader peptide gene tnaC was used to control the tetracycline resistance gene tetA [16]. For the TrpRPmtr-hipA system, Pmtr (promoter of E. coli gene mtr), was used to control the expression of a toxin gene hipA [21]. The resulting strains BTS (tnaC-tetA) and BTS1 (TrpR-Pmtr-hipA) containing these two systems have
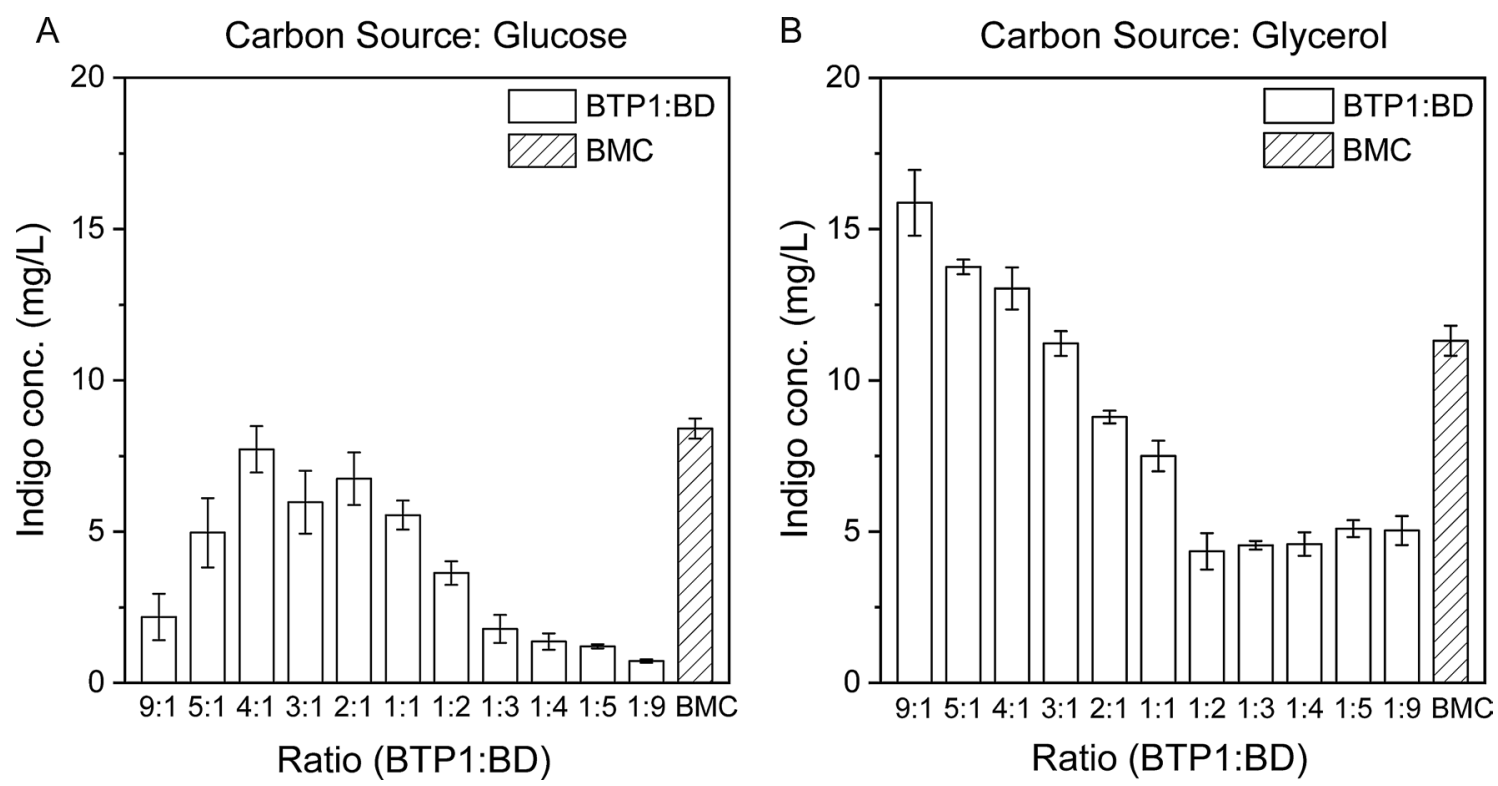

Fig. 2 Comparison of the indigo bioproduction by the BMC mono-culture and BTP1:BD co-culture inoculated at different ratios using $\mathbf{A}$ glucose and $\mathbf{B}$ glycerol as the carbon substrate. The error bars represent the standard error of at least three biological replicates 
been previously characterized [16, 21], and for this work, they were co-cultivated with the downstream strain BD for the indigo biosynthesis, respectively.

As shown in Fig. 3, the indigo production on glycerol were significantly improved when tnaC-tetA (Fig. 3A) or TrpR-Pmtr-hipA (Fig. 3B) were recruited. In the BTS:BD system (containing tnaC-tet $A$ ), the highest indigo production was $47.7 \mathrm{mg} / \mathrm{L}$ at the inoculation ratio of $2: 1$. The highest indigo production by the BTS1:BD co-culture system (containing TrpR-Pmtr-hipA) reached $55.4 \mathrm{mg} / \mathrm{L}$ at the inoculation ratio of $5: 1$, which is $248 \%$ higher than the BTP1:BD co-culture without the biosensor-assisted cell selection system. The use of the biosensors in the mono-culture controls showed a different effect on the indigo production. The indigo concentrations of the engineered mono-culture strains BMS and BMS1 were $10.6 \mathrm{mg} / \mathrm{L}$ and $5.9 \mathrm{mg} / \mathrm{L}$, respectively, both of which are lower than the BMC strain without any cell selection system. This result implied that the biosensor-assisted cell selection system may not be helpful for the biosynthesis in monocultures, which is consistent with the previous findings $[16,18]$.

Furthermore, we investigated the biosynthesis performance of the BTS1:BD co-culture in a series of medium containing different glucose/glycerol ratios. As shown in Fig. 4, when glucose was the sole carbon source, the indigo production was only $14.1 \mathrm{mg} / \mathrm{L}$. The increase of the glycerol content in the substrate gradually improved the production. The indigo concentration was increased

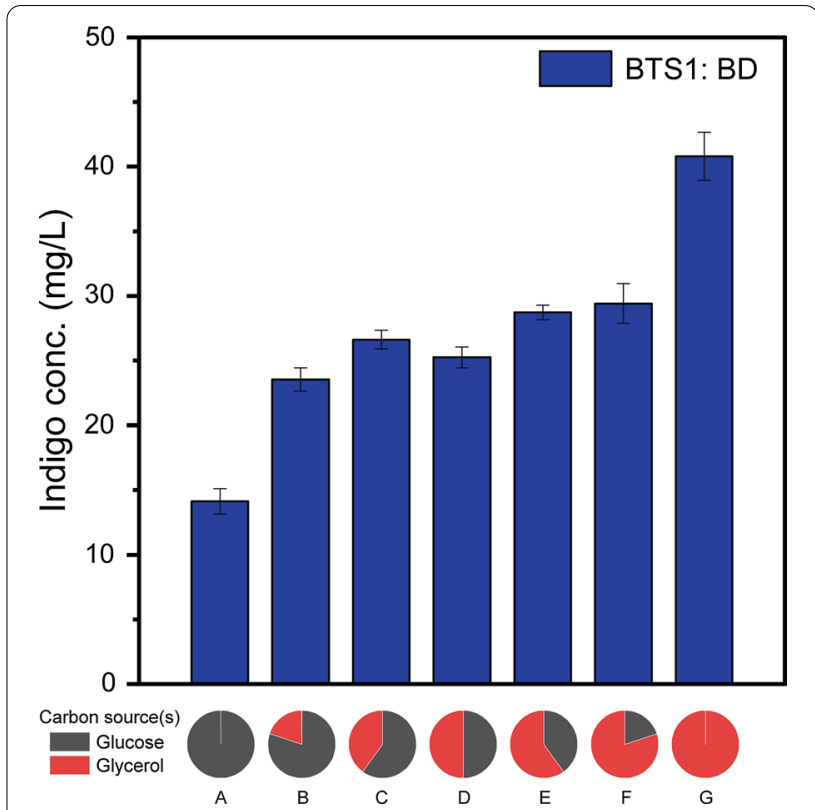

Fig. 4 Comparison of the indigo bioproduction by the BTS1:BD co-culture grown on (A) $5 \mathrm{~g} / \mathrm{L}$ glucose, (B) $4 \mathrm{~g} / \mathrm{L}$ glucose and $1 \mathrm{~g} / \mathrm{L}$ glycerol, (C) $3 \mathrm{~g} / \mathrm{L}$ glucose and $2 \mathrm{~g} / \mathrm{L}$ glycerol, (D) $2.5 \mathrm{~g} / \mathrm{L}$ glucose and $2.5 \mathrm{~g} / \mathrm{L}$ glycerol, (E) $2 \mathrm{~g} / \mathrm{L}$ glucose and $3 \mathrm{~g} / \mathrm{L}$ glycerol, (F) $1 \mathrm{~g} / \mathrm{L}$ glucose and $4 \mathrm{~g} / \mathrm{L}$ glycerol, and (G) $5 \mathrm{~g} / \mathrm{L}$ glycerol. The co-culture strains were inoculated at the 1:1 ratio. The error bars represent the standard error of at least three biological replicates

to $40.8 \mathrm{mg} / \mathrm{L}$ when glycerol was the sole carbon source, which is $190 \%$ higher than the production on glucose only. The results hereby are consistent with the previous
A

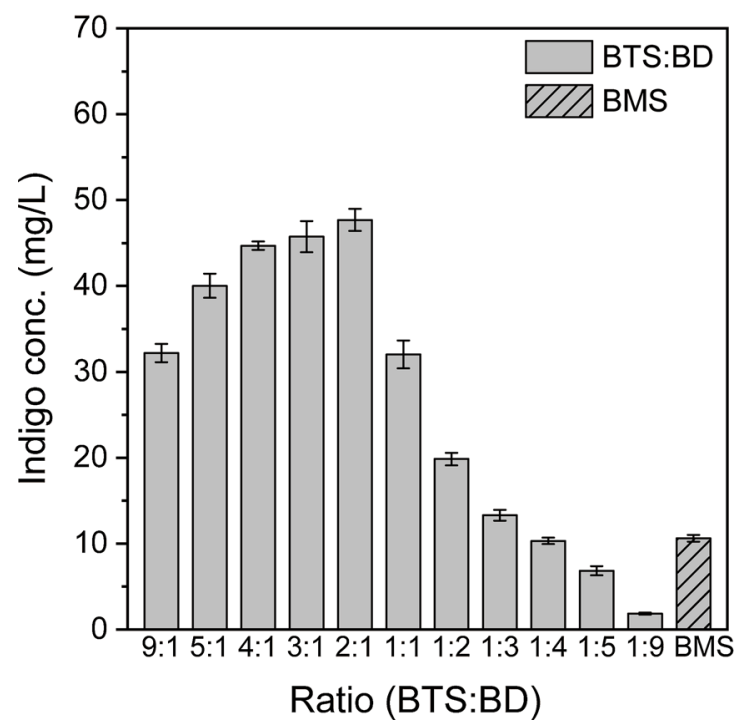

B

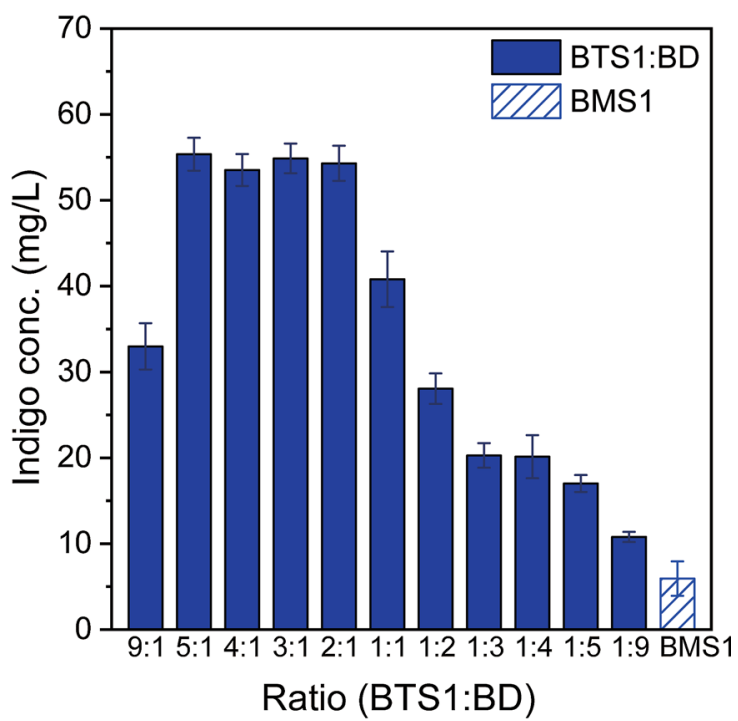

Fig. 3 Comparison of the indigo bioproduction on glycerol using different biosensor-assisted cell selection systems. A The indigo production by the BTS:BD co-culture and the BMS mono-culture containing the thaC-tetA system. B The indigo production by the BTS1:BD co-culture and the BMS1 mono-culture containing the Pmtr-hipA system. The error bars represent the standard error of at least three biological replicates 
section and indicate that glycerol is a preferred carbon substrate for the indigo biosynthesis. As such, glycerol was adopted for the following experiments.

\section{Using global transcription machinery engineering (gTME) to strengthen the co-culture's biosynthesis performance} Next, we sought to promote the biosynthetic capability of the downstream strain in the context of the co-culture. To this end, we adopted a novel approach based on global transcription machinery engineering (gTME) to alter the cellular transcriptome and introduce phenotypic diversity. A facile colorimetric assay was then utilized to identify the mutants with desired biosynthesis enhancement. The schematic design of this experiment is shown in Fig. 5. Specifically, E. coli gene rpoA encoding the $\alpha$ subunit of the RNA polymerase was randomly mutated through replication in E. coli XL1Red which is capable of in vivo DNA mutagenesis. A rpo $A$ mutants' library was thus created and introduced into the downstream strain, generating 409 variants. Each of these downstream strain variants expressed a particular rpoA mutant and was then co-cultured with the upstream strain BTR1 at 1:1 inoculation ratio in 96-well plates. A colorimetric assay was used to rapidly screen the co-cultures to identify the ones with improved indigo production. Notably, since indigo has a distinctive blue color, high-performing co-culture variants can be distinguished by monitoring the blue color intensity in a high throughput manner.

The statistical analysis of the biosynthesis performance difference between the co-culture variants grown in 96-well plate was shown in Fig. 6A. Most variants (303 of 409) presented a lower productivity compared to the control group, 106 variants exhibited improvement in the indigo bioproduction (which is about $25 \%$ of total mutations), indicating that this mutation strategy is efficient. 9 variants showed significant improvement with the indigo production fold change higher than 3. Top five variants (the indigo concentration greater than $12 \mathrm{mg} / \mathrm{L}$ in 96-wells plate) were chosen to verify their capability by co-cultured with the upstream strain BTR1 in the culture tubes. As shown in Fig. 6B, all top five mutants showed higher production performance than the control co-culture BTR1:BRC expressing the wild-type rpoA. The best co-culture variant, BTR1: BRM-353 (harboring the $r p o A^{353}$ mutant), produced $80.6 \mathrm{mg} / \mathrm{L}$ indigo from $5 \mathrm{~g} / \mathrm{L}$ glycerol after $48 \mathrm{~h}$ cultivation, which was $135 \%$ higher than the BTR1:BRC control co-culture. These findings suggested that desirable phenotypes for high indigo biosynthesis in the co-culture were successfully created using gTME. To our knowledge, this is the first report utilizing gTME in the context of microbial co-cultures,

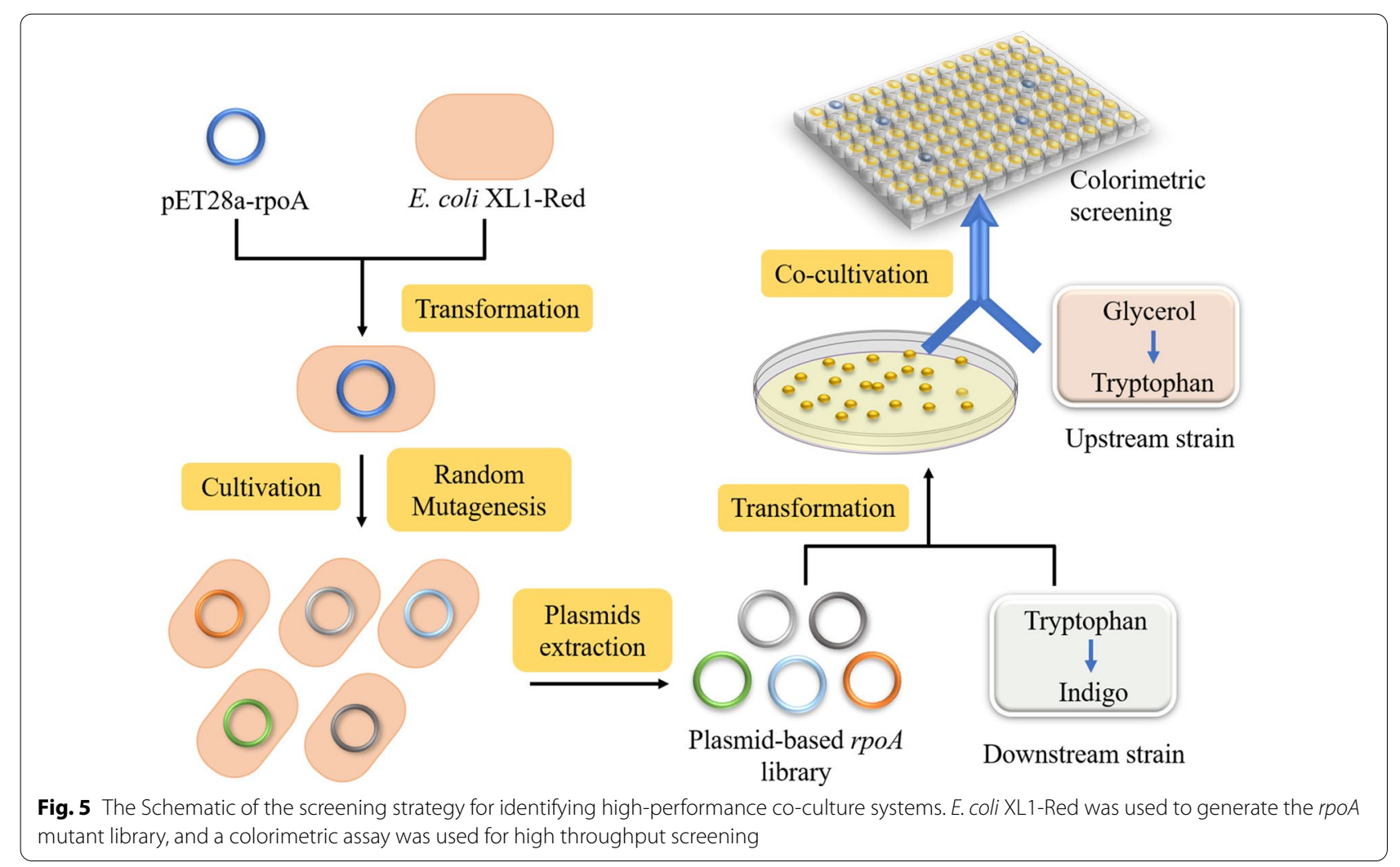



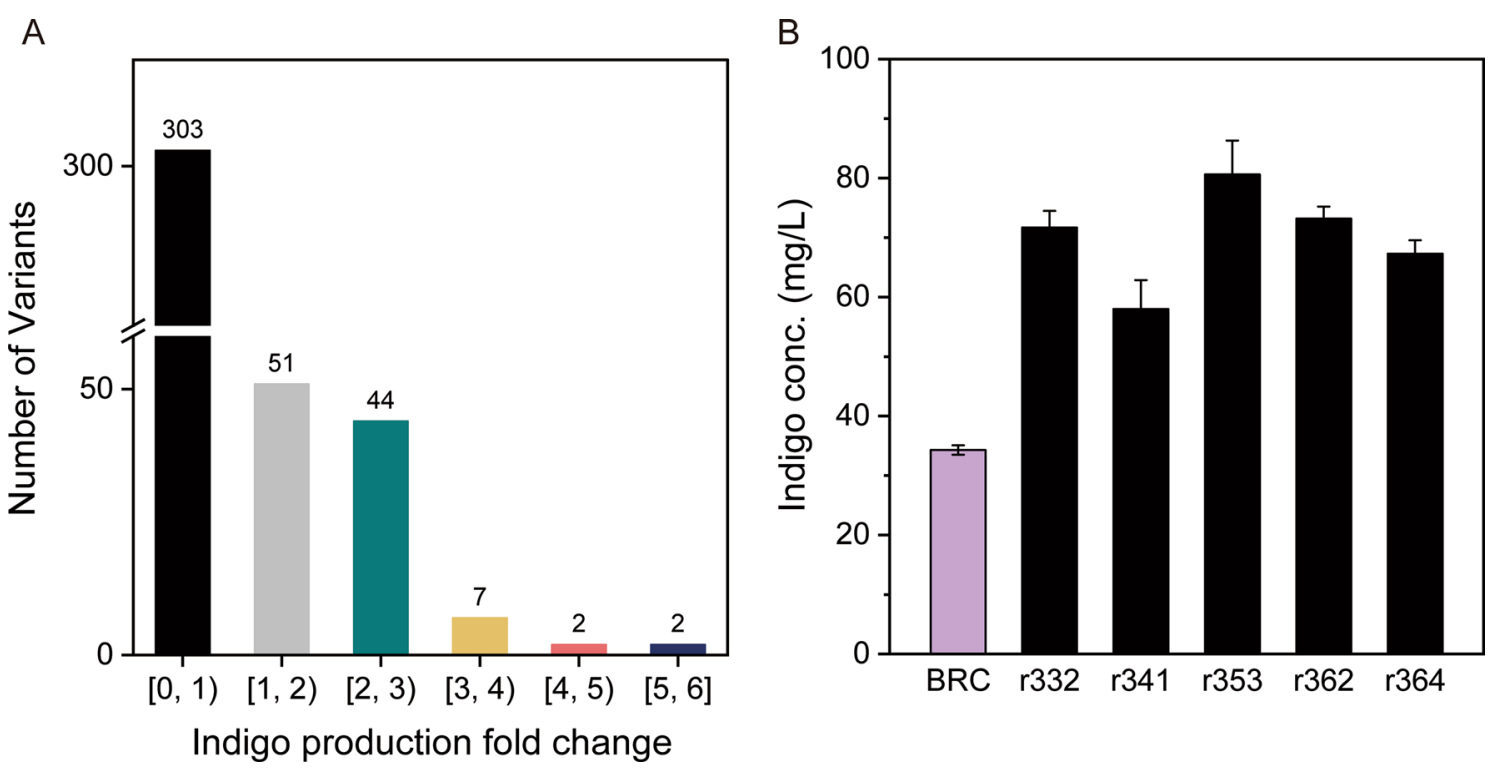

Fig. 6 The indigo bioproduction by the co-cultures containing the rpoA mutants. A The statistical analysis of the biosynthesis performance difference between the co-culture variants grown in 96-well plate. B Comparison of the indigo production by the top five co-culture variants. The upstream and downstream strains were inoculated at 1:1 ratio. The error bars represent the standard error of at least three biological replicates

offering a new research avenue to advance microbial coculture engineering.

\section{Characterization of the identified downstream strain mutant}

To characterize the $r p o A^{353}$ mutant, its DNA sequence was analyzed. It was found that there are more than 700point mutations in both the promoter and coding region of the $r p o A^{353}$ mutant, which accounts for more than $50 \%$ of the original rpoA gene. This clearly indicates that the DNA mutagenesis method using the XL1-Red strain is highly efficient. Since $r p o A$ is a key component for the global transcription, such a high degree of the mutation is considered to create strong perturbations for the cellular behaviors.

Strain BRM-353 harboring the $r p o A^{353}$ mutant was characterized for its growth and tryptophan bioconversion capability. Specifically, BRM-353 and BRC (control strain harboring the wild-type $r p o A$ gene) were both fed with $500 \mathrm{mg} / \mathrm{L}$ exogenous tryptophan and cultivated as mono-cultures at $30{ }^{\circ} \mathrm{C}$. It was found that the mutant strain did not display any significant difference in the growth pattern compared with the control strain BRC (Fig. 7A). Moreover, the dynamic changes of tryptophan and indigo concentrations over time were monitored for $48 \mathrm{~h}$. As shown in Fig. 7A, tryptophan in both BRC and BRM-353 cultures was quickly depleted after $12 \mathrm{~h}$, and their consumption rates followed a similar pattern. Interestingly, these two strains showed different indigo production behavior. The indigo concentration in the BRM-353 culture increased rapidly $3 \mathrm{~h}$ after the inoculation and plateaued at around $101 \mathrm{mg} / \mathrm{L}$ after $24 \mathrm{~h}$, whereas the control strain's indigo biosynthesis peaked at around $50 \mathrm{mg} / \mathrm{L}$. The results hereby confirm that the mutant strain BRM-353 possesses strong capability of producing indigo from tryptophan.

Next, the indigo biosynthesis by the identified BTR1:BRM-353 co-culture was optimized by varying the inoculation ratio to coordinate the biosynthetic strengths of the upstream and downstream pathway modules. As shown in Fig. 7B, for most of the tested inoculation ratios, the indigo production was significantly higher than the control co-culture BTR1:BRC using the wild type rpoA. The highest indigo concentration of $104.3 \mathrm{mg} / \mathrm{L}$ was achieved at the inoculation ratio of $2: 1$, which is $204 \%$ higher than the optimum of the BTR1:BRC co-culture and $1142 \%$ higher than the starting indigo-producing strain. The result confirmed the effectiveness of gTME in co-culture engineering. More importantly, it reveals that, even when the biosynthetic pathway is not further modified, the metabolic status of a co-culture strain can be altered by engineering strategies such as gTME to trigger the biosynthesis enhancement.

We also compared the growth profiles of the co-cultures before and after the introduction of the $r p o A^{353}$ mutant. The strain-to-strain ratio within both co-cultures' populations (Additional file 1: Fig. S3) only fluctuated to a small degree throughout the cultivation, 

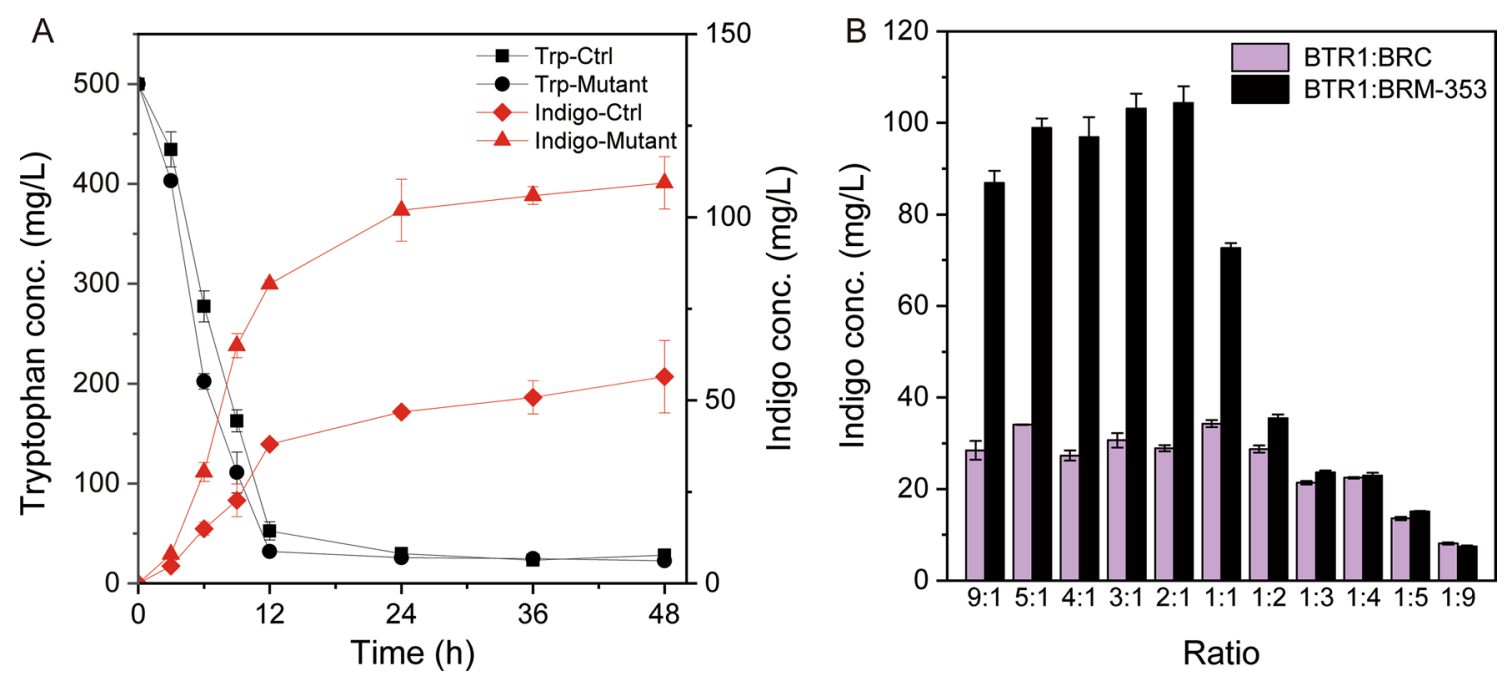

Fig. 7 Characterization of BRM-353 mutant's biosynthesis capability. A Time profiles of the tryptophan consumption and indigo production by BRC (control) and BRM-353 (mutant) fed with exogenous tryptophan. B The effect of the inoculation ratio on the indigo production by BTR1:BRC and BTR1:BRM-353 co-cultures. The error bars represent the standard error of at least three biological replicates

indicating the stability of the co-culture system. There are no significant differences between the two co-cultures' growth profiles, which suggests that gTME did not change the interaction between the co-culture strains for their co-growth behavior.

\section{Discussion}

Glucose is a commonly used carbon source for microbial biosynthesis [30, 31], while our study shows that glycerol is a preferred carbon source for the indigo biosynthesis. It has been found that the expression and activity of TnaA, a key pathway enzyme, is inhibited by glucose [32]. As a result, the use of glucose limited the formation of the pathway intermediate indole, leading to the low indigo production on glucose. The use of glycerol avoided such inhibition on TnaA and caused higher tryptophan conversion and indigo production.

On the other hand, the integration of the biosensor-assisted cell selection system into the upstream strain helped eliminate the low-performing cells of the upstream strain's subpopulation and thus improved the tryptophan provision for the indigo production. The two tested biosensor systems showed comparable performance for the indigo biosynthesis improvement. However, the use of TrpR-Pmtr-hipA system did not require the addition of any exogenous antibiotic for cell selection, which is considered advantageous for process economy, especially for applications in larger scale process. Notably, this strategy requires TrpR (encoded by trpR on the chromosome) as the sensor protein. The tnaC-tetA system, however, does not need additional sensor proteins for the sensing mechanism to work. Both systems have their advantages and have been utilized for facilitating microbial biosynthesis in previous studies [16, 21].

gTME has been demonstrated to be an effective technique for introducing diverse changes into the global metabolic network [20,33]. Previous gTME studies have been mainly pursued using the mono-culture of a single microbial strain. In this work, we implemented gTME within the context of a microbial co-culture, which expands the application of this powerful approach. Different from the conventional approach using error-prone PCR to carry out random DNA mutagenesis, this study recruited $E$. coli XL1-RED with hyper mutation efficiency for generating rpoA gene mutants in vivo. The resulting rpo $A$ mutant genes, once introduced into the downstream strain, changed its global transcriptome and diversified the phenotypes, some of which are helpful for the indigo production in the context of the co-culture. For the best mutant $r p o A^{353}, 50 \%$ of the gene sequence is different from the wild type rpoA. As such, it is highly challenging to track which specific mutations caused the phenotypic changes. Notably, the characterization of the identified mutant showed that its biosynthesis improvement can be attributed to the enhanced tryptophan-toindigo conversion capability (Fig. 7A) and its growth behavior was still similar to the wild type strain (Additional file 1: Fig. S4). It is postulated that such enhancement is because the rpoA mutation downregulated of the competing pathway(s) in the background metabolism or altered cellular environment that better supports the pathway enzymes' in vivo activities. However, how 
exactly the transcriptomic changes induced by $r p o A^{353}$ contribute to such enhancement remains unknown and needs to be further investigated.

Traditional mutant library screening methods are also based on the use of mono-cultures. For example, a library of mutant strains is fed with exogenous metabolite precursors for identification of individual mutants with high conversion. However, it is possible that the identified mutants may not co-grow well with the upstream strain in the co-culture setting, resulting in negative screening results. For this study, the screening of the mutant library was conducted using the co-cultures, i.e. by co-cultivating the downstream strain mutants with the upstream strain. Such a screening method considers the metabolic interaction between the co-culture strains for completing the indigo biosynthesis, which eliminated the issue associated with mono-culture-based approach and thus improved the screening process efficiency. To this end, the results of this work lay a foundation for using similar strategies for engineering other microbial co-cultures to improve biosynthesis performance in future studies.

\section{Conclusion}

In this work, we combined several metabolic engineering strategies to establish and improve the indigo biosynthesis in E. coli. Glycerol was found to be a better carbon substrate than glucose, and employment of modular coculture engineering strategies resulted in superior production performance over the mono-culture approach. On top of these efforts, biosensor-assisted cell selection was introduced to the upstream co-culture strain to strengthen the tryptophan provision, whereas global transcription machinery engineering was adopted to enhance the downstream strain's capability for converting tryptophan to indigo. When integrated together, these strategies led to the production of $104.3 \mathrm{mg} / \mathrm{L}$ indigo, 11.4-fold higher than that of the starting $E$. coli strain.

\section{Materials and methods}

\section{Plasmids and strains construction}

Plasmids and strains used in this work are listed in Tables 1 and 2. Flavin-containing monooxygenase (FMO) from Methylophaga aminisulfidivorans was codonoptimized and synthesized by Bio basic Inc. Restriction enzymes, DNA ligase, and DNA polymerase were purchased from New England Biolabs. Plasmid extraction kit and DNA purification kit were purchased from Zymo Research. Plasmid construction was conducted using DH5 $\alpha$ competent cells (New England Biolabs), and mutation was performed by using XL1-Red cells (Agilent). Sequences of the primers used in this study are listed in Additional file 1: Table S1.

To construct plasmid pDF, the fmo gene from Methylophaga aminisulfidivorans was codon-optimized and synthesized by Bio Basic Inc. The fmo gene was digested by NdeI and XhoI then ligated with pET28a treated with the same enzymes. To construct plasmid pMF, the fmo gene was digested by NdeI and XhoI then ligated with pCDFDuet-1 treated with the same enzymes.

For the construction of plasmid pDT, the DNA fragment containing promoter Ptet and tetracycline resistance gene tet $A$ was PCR amplified from plasmid pBR322 using primers tet- $\mathrm{F}$ and tet- $\mathrm{R}$, then inserted into plasmid pET21c using the EcoRI and XhoI sites.

Table1 Plasmids used in this study

\begin{tabular}{|c|c|c|}
\hline Plasmid & Description & Source \\
\hline pET28a & T7 promoter, Kan ${ }^{R}$ & Novagen \\
\hline pACYCDuet-1 & Double T7 promoters, $\mathrm{Cm}^{\mathrm{R}}$ & Novagen \\
\hline pET21c & T7 promoter, Amp ${ }^{R}$ & Novagen \\
\hline pCDFDuet-1 & T7 promoter, Strp ${ }^{R}$ & Novagen \\
\hline pTE3 & $\begin{array}{l}\text { pET28a carrying the trpE } E^{f b r} \text { and } a r o G^{f b r} \text { genes under the control of the constitutive Zymomonas mobilis pyruvate } \\
\text { decarboxylase promoter (Ppdc) }\end{array}$ & [21] \\
\hline pTD2 & $\begin{array}{l}\text { pACYC Duet carrying the } \operatorname{trp} D, \operatorname{trp} C, \operatorname{trp} B \text { and trpA genes under the control of the constitutive Zymomonas mobilis } \\
\text { pyruvate decarboxylase promoter (Ppdc) }\end{array}$ & {$[21]$} \\
\hline pSE & pET21c carrying tryptophan regulatory element (tnaC operon) and the tet $A$ gene, $A m p^{R}$ & [16] \\
\hline pSE1 & pET21c carrying the hipA gene under the control of the mtr promoter (Pmtr) & [21] \\
\hline pDF & pET28a carrying the fmo gene (from Methylophaga aminisulfidivorans) under control of T7 promoter & This study \\
\hline pMF & pCDFDuet-1 carrying the fmo gene (from Methylophaga aminisulfidivorans) under control of T7 promoter & This study \\
\hline pDT & pET21c carrying the tetA gene under the control of the tet $A$ promoter (Ptet) & This study \\
\hline pTSO & pET28a carrying the proA promoter with the kan $R$ gene replaced by the strpR gene & This study \\
\hline pTS1 & pTSO carrying rpoA gene under control of proA promoter & This study \\
\hline pTS2 & pTS1 after random mutagenesis & This study \\
\hline
\end{tabular}


Table 2 Strains used in this study

\begin{tabular}{|c|c|c|}
\hline Strain & Description & Source \\
\hline $\mathrm{BH} 2$ & E. coli BL21(DE3) $\triangle x y \mid A \Delta t y r A \Delta p h e A$ & [22] \\
\hline XL1-Red & $\begin{array}{l}\left.\text { F-endA1 gyrA96(nal }\left.\right|^{R}\right) \text { thi-1 relA1 lac glnV44 } \\
\left.\text { hsdR17( } \mathrm{r}_{\mathrm{K}}^{-} \mathrm{m}_{\mathrm{K}}^{+}{ }^{+}\right) \text {mutS mutT mutD5 } \operatorname{Tn} 10\end{array}$ & Agilent \\
\hline BTP1 & $\mathrm{BH} 2$ harboring pTE3, pTD2 and pET21c & [21] \\
\hline BTS & BH2 harboring pTE3, pTD2 and pSE & [16] \\
\hline BTS1 & $\mathrm{BH} 2$ harboring pTE3, pTD2 and pSE1 & {$[21]$} \\
\hline BTR1 & BTS1 harboring pTS & This study \\
\hline BF & $\mathrm{BH} 2$ harboring $\mathrm{pDF}$ & This study \\
\hline BD & $\mathrm{BH} 2$ harboring pACYCDuet-1, pDT and pDF & This study \\
\hline BRC & BD harboring pTS1 & This study \\
\hline BRM & BD harboring pTS2 & This study \\
\hline BRM-353 & $\mathrm{BD}$ harboring pTS2 with the $r p o \mathrm{~A}^{353}$ mutant & This study \\
\hline $\mathrm{BMC}$ & BTP1 harboring pMF & This study \\
\hline BMS & BTS harboring pMF & This study \\
\hline BMS1 & BTS1 harboring pMF & This study \\
\hline XLR & XL1-Red harboring pTS1 & This study \\
\hline
\end{tabular}

To construct plasmid pTS0, the DNA fragment containing promoter Pstrp and streptomycin resistance gene strpR was PCR amplified from plasmid pCDFDuet-1 using primers strp-F and strp-R. Using the EcoNI site, the PCR product was inserted into plasmid pET28a-PproA containing a weak constitutive proA promoter [34].

For the construction of plasmid pTS1, the rpoA gene was PCR amplified from the genome of E. coli K12 chromosome by primers rpoA-F and rpoA-R. The $r p o A$ gene was digested by SpeI and XhoI then ligated with pTS0 treated with the same enzymes to generate pTS1.

To construct the mutation library of pTS2, pTS1 was transformed into XL1-Red for ten cultivation generations, the mutation pTS2 library was generated by plasmid extraction of the 10th cultivation.

\section{Medium and cultivation}

For the production of indigo, strains were cultured in M9 special medium. One liter of M9 special medium contained 5 g glucose or glycerol, $0.5 \mathrm{~g}$ yeast extract, $8.5 \mathrm{~g}$ $\mathrm{Na}_{2} \mathrm{HPO}_{4} \cdot 2 \mathrm{H}_{2} \mathrm{O}, 3 \mathrm{~g} \mathrm{K \textrm {H } _ { 2 }} \mathrm{PO}_{4}, 1 \mathrm{~g} \mathrm{NH} \mathrm{N}_{4} \mathrm{Cl}, 0.5 \mathrm{~g} \mathrm{NaCl}$, $40 \mathrm{mg}$ tyrosine, $40 \mathrm{mg}$ phenylalanine and trace elements. Trace elements included $0.4 \mathrm{mg} / \mathrm{L} \mathrm{Na}{ }_{2}$ EDTA, $0.03 \mathrm{mg} / \mathrm{L}$ $\mathrm{H}_{3} \mathrm{BO}_{3}, 1 \mathrm{mg} / \mathrm{L}$ thiamine, $0.94 \mathrm{mg} / \mathrm{L} \mathrm{ZnCl}_{2}, 0.5 \mathrm{mg} / \mathrm{L}$ $\mathrm{CoCl}_{2}, 0.38 \mathrm{mg} / \mathrm{L} \mathrm{CuCl}$, $1.6 \mathrm{mg} / \mathrm{L} \mathrm{MnCl}_{2}, 3.77 \mathrm{mg} / \mathrm{L}$ $\mathrm{CaCl}_{2}$, and $3.6 \mathrm{mg} / \mathrm{L} \mathrm{FeCl}_{2}$.

All strains were pre-cultured overnight in LB medium with proper antibiotics at $37{ }^{\circ} \mathrm{C}, 250 \mathrm{rpm}$. For the mono-culture experiment, $2 \%(\mathrm{v} / \mathrm{v})$ overnight LB cultures of the desired $E$. coli strains were inoculated in fresh LB medium with proper antibiotics for $6 \mathrm{~h}$. Then cells were collected and re-suspended in M9 special medium containing appropriate antibiotics and $0.1 \mathrm{mM}$ IPTG with an initial $\mathrm{OD}_{600}$ of 0.6. After cultivation at $30{ }^{\circ} \mathrm{C}$ for $48 \mathrm{~h}$, samples were collected for analysis. For the co-culture experiment, $2 \%(\mathrm{v} / \mathrm{v})$ overnight LB cultures of the desired upstream and downstream strains were inoculated in fresh LB medium for $6 \mathrm{~h}$, respectively. Both upstream and downstream strains were collected and re-suspended in M9 special medium containing appropriate antibiotics and $0.1 \mathrm{mM}$ IPTG. For the culture tube scale co-culture, the upstream and downstream strains were inoculated into $2 \mathrm{~mL}$ fresh $\mathrm{M} 9$ special medium with a series of desired ratios to reach an initial total $\mathrm{OD}_{600}$ of 0.6 . For the 96 well plate scale co-culture, the upstream and downstream strains were inoculated into $200 \mu \mathrm{L}$ M9 special medium in each well to reach an initial total $\mathrm{OD}_{600}$ of 0.6 . The plates were sealed by aluminum sealing film (Platemax, Axygen). After cultivation at $30{ }^{\circ} \mathrm{C}$ and $250 \mathrm{rpm}$ for $48 \mathrm{~h}$, samples were collected for analysis.

For characterization of the downstream strain mutant BRM-353, both control strain and mutant strain were cultivated in M9 special medium (containing $5 \mathrm{~g} / \mathrm{L}$ of glycerol) with additional $500 \mathrm{mg} / \mathrm{L}$ tryptophan. The $\mathrm{OD}_{600}$ value, the tryptophan concentration, and the indigo concentration at desired time points were measured.

To distinguish the upstream and downstream strains in the co-culture, the co-culture samples were collected, diluted with sterile water, and spread onto a set of LB agar plates containing kanamycin, streptomycin, and chloramphenicol. After overnight incubation, the colonies on the plates were transferred to a new set of LB agar plates containing tetracycline. Since the downstream strain is resistant to tetracycline and the upstream strain is not, the colonies that could grow on both sets of plates were counted as the downstream strains. The ratio between the colony numbers grown on the two sets of plates represents the ratio of the co-culture strains' populations in the culture.

\section{Mutation of the $r p o A$ gene}

The plasmid pTS1 containing the wild-type $r p o A$ gene was transformed into $E$. coli XL1-Red, resulting strain XLR. XLR was cultured in LB medium with $50 \mathrm{mg} / \mathrm{L}$ streptomycin at $37{ }^{\circ} \mathrm{C}, 250 \mathrm{rpm}$ for $12 \mathrm{~h}$, and the resulting culture was transferred into fresh LB medium with streptomycin $(2 \% \mathrm{v} / \mathrm{v})$. After ten generations of cultivation, a library of plasmid pTS1 mutants was generated and transformed into strain BD, yielding strain BRM. All BRM colonies were selected and characterized for the indigo biosynthesis by co-cultivation with the upstream strain BTR1. 


\section{Extraction of indigo}

The cell cultures were centrifuged at $5000 \mathrm{rpm}$ for 10 min to collect the cell pellet. The precipitate was a mixture of cells, cell-bound indigo, and free indigo particles. After re-suspended in DMSO, mixture was sonicated on ice for 30 cycles; each cycle comprised 1-s sonication and 6-s break. The sonicated mixture was then centrifuged at 10,000 rpm for $5 \mathrm{~min}$, and supernatant was collected for indigo analysis. The extraction process was repeated until the supernatant is free of blue color.

\section{Metabolite analysis}

Pathway metabolites analysis were performed by high performance liquid chromatography using a Shimadzu Prominence HPLC System equipped with UV detector SPD-20AV. For tryptophan quantification, $1 \mathrm{~mL}$ of cell culture was centrifuged at 10,000 rpm for $5 \mathrm{~min}$, and the supernatant was filtered through $0.45 \mu \mathrm{m}$ PVDF membrane syringe filters (Whatman Inc). $10 \mu \mathrm{L}$ of filtered sample was injected into a ZORBAX Eclipase Plus C18 column $(4.6 \times 150 \mathrm{~mm}, 5 \mu \mathrm{m}$, Agilent Technologies) and eluted by solvent $\mathrm{A}$ ( $0.5 \%$ acetic acid) and solvent B (100\% acetonitrile). The total flow rate was maintained at $0.6 \mathrm{~mL} / \mathrm{min}$, and the following gradient was used for tryptophan separation: $0-10 \mathrm{~min}$, a linear gradient of B from 0 to $50 \%$; $10-10.2 \mathrm{~min}$, a linear gradient of B from 50 to $100 \%$; $10.2-13 \mathrm{~min}, 100 \% \mathrm{~B}$; 13.1 min, a linear gradient of $B$ from 100 to $0 \%$; $13.1-$ $16 \mathrm{~min}, 0 \%$ B (all in vol\%). Absorption at $280 \mathrm{~nm}$ was monitored for the tryptophan quantification.

For the indigo quantification, $1 \mathrm{~mL}$ extraction mixture was centrifuged at $10,000 \mathrm{rpm}$ for $5 \mathrm{~min}$, and the supernatant was filtered through $0.45 \mu \mathrm{m}$ PVDF membrane syringe filters. $10 \mu \mathrm{L}$ of filtered sample was injected into a Hamilton PRP-C18 column $(4.6 \times 150 \mathrm{~mm}, 5 \mathrm{~mm}$, Hamilton, USA) and eluted by solvent A (0.5\% acetic acid) and solvent $B$ (100\% acetonitrile). The total flow rate was maintained at $0.6 \mathrm{~mL} / \mathrm{min}$, and the following gradient was used: $0-5 \mathrm{~min}$, a linear gradient of $\mathrm{B}$ from 60 to $100 \%$; 5-20 min, a linear gradient of B from 100 to $60 \%$ (all in vol\%). Absorption at $620 \mathrm{~nm}$ was monitored for the indigo quantification.

\section{Supplementary Information}

The online version contains supplementary material available at https://doi. org/10.1186/s12934-021-01636-w.

Additional file 1. Table S1. Sequences of the primers used in this study. Fig. S1. Comparison of indigo production at different temperatures. Strain BD containing the fmo gene was fed with $100 \mathrm{mg} / \mathrm{L}$ of tryptophan or indole for the bioproduction. The error bars represent the standard error of at least three biological replicates. Fig. S2. Schematic illustration of cell selection using (A) TrpR-Pmtr-hipA and (B) tnaC-tetA systems. Fig. S3. The strain-to-strain ratio change over time within the populations of (A) the BTR1:BRC co-culture and (B) BTR1:BRM-353 co-culture. Fig. S4. Growth curves of the downstream strains BRC (control) and BRM-353 (mutation). Both strains were cultured in shake flask with M9 medium containing 5 g/L glycero

\section{Acknowledgements}

Not applicable.

\section{Authors' contributions}

$T C, X W, L Z$, and AS performed the experiments; TC, XW, and $H Z$ conceived and designed the project; $\mathrm{TC}$ analyzed the primary data; $\mathrm{HZ}$ and $\mathrm{YL}$ supervised the project. TC, XW, LZ, YL, and $\mathrm{HZ}$ reviewed the manuscript. All authors read and approved the final manuscript.

\section{Funding}

This work is supported by the startup research fund from Rutgers, The State University of New Jersey. Tingting Chen is a recipient of the CSC Ph.D. fellowship.

\section{Availability of data and materials}

The datasets used and/or analyzed during the current study are available from the corresponding author on reasonable request.

\section{Declarations}

Ethics approval and consent to participate

Not applicable.

\section{Consent of publication}

Not applicable.

\section{Competing interests}

The authors declare that they have no competing interests.

\section{Author details}

'Department of Chemical and Biochemical Engineering, College of Chemistry and Chemical Engineering, Xiamen University, Xiamen 361005, Fujian, China. ${ }^{2}$ Department of Chemical and Biochemical Engineering, Rutgers, The State University of New Jersey, Piscataway, NJ 08854, USA. ${ }^{3}$ School of Arts and Sciences, Rutgers, The State University of New Jersey, 77 Hamilton Street, New Brunswick, NJ 08901, USA. ${ }^{4}$ The Key Lab for Synthetic Biotechnology of Xiamen City, Xiamen University, Xiamen 361005, Fujian, China. ${ }^{5}$ Fujian Collaborative Innovation Center for Exploitation and Utilization of Marine Biological Resources, Xiamen University, Xiamen 361005, Fujian, China.

Received: 4 June 2021 Accepted: 16 July 2021

Published online: 04 August 2021

\section{References}

1. Stasiak N, Kukula-Koch W, Glowniak K. Modern industrial and pharmacological applications of indigo dye and its derivatives-a review. Acta Pol Pharm. 2014;71:215-21.

2. Han GH, Gim GH, Kim W, Seo SI, Kim SW. Enhanced indirubin production in recombinant Escherichia coli harboring a flavin-containing monooxygenase gene by cysteine supplementation. J Biotechnol. 2013;164:179-87.

3. Meyer A, Wursten M, Schmid A, Kohler HPE, Witholt B. Hydroxylation of indole by laboratory-evolved 2-hydroxybiphenyl 3-monooxygenase. J Biol Chem. 2002;277:34161-7.

4. Qu YY, Pi WQ, Ma F, Zhou JT, Zhang XW. Influence and optimization of growth substrates on indigo formation by a novel isolate Acinetobacter sp. PP-2. Bioresour Technol. 2010;101:4527-32.

5. Qu YY, Zhang XW, Ma Q, Ma F, Zhang Q, Li XL, et al. Indigo biosynthesis by Comamonas sp. MQ Biotechnol Lett. 2012;34:353-7. 
6. Honda K, Yamashita S, Nakagawa H, Sameshima Y, Omasa T, Kato J, et al. Stabilization of water-in-oil emulsion by Rhodococcus opacus B-4 and its application to biotransformation. Appl Microbiol Biotechnol. 2008;78:767-73.

7. Doukyu N, Toyoda K, Aono R. Indigo production by Escherichia coli carrying the phenol hydroxylase gene from Acinetobacter sp. strain ST-550 in a water-organic solvent two-phase system. Appl Microbiol Biotechnol. 2003;60:720-5.

8. Berry A, Dodge TC, Pepsin M, Weyler W. Application of metabolic engineering to improve both the production and use of biotech indigo. J Ind Microbiol Biotechnol. 2002;28:127-33.

9. Ensley BD, Ratzkin BJ, Osslund TD, Simon MJ, Wackett LP, Gibson DT. Expression of naphthalene oxidation genes in Escherichia coli results in the biosynthesis of indigo. Science. 1983;222:167-9.

10. Choi HS, Kim JK, Cho EH, Kim YC, Kim JI, Kim SW. A novel flavin-containing monooxygenase from Methylophaga sp. strain SK1 and its indigo synthesis in Escherichia coli. Biochem Biophys Res Commun. 2003;306:930-6.

11. Du J, Yang D, Luo ZW, Lee SY. Metabolic engineering of Escherichia coli for the production of indirubin from glucose. J Biotechnol. 2018;267:19-28.

12. Han GH, Bang SE, Babu BK, Chang M, Shin HJ, Kim SW. Bio-indigo production in two different fermentation systems using recombinant Escherichia coli cells harboring a flavin-containing monooxygenase gene (fmo). Process Biochem. 2011;46:788-91.

13. Murdock D, Ensley BD, Serdar C, Thalen M. Construction of metabolic operons catalyzing the de novo biosynthesis of indigo in Escherichia coli. Bio-Technology. 1993;11:381-6.

14. Chen T, Zhou Y, Lu Y, Zhang H. Advances in heterologous biosynthesis of plant and fungal natural products by modular co-culture engineering. Biotechnol Lett. 2019;41:27-34.

15. Roell GW, Zha J, Carr RR, Koffas MA, Fong SS, Tang YJ. Engineering microbial consortia by division of labor. Microb Cell Fact. 2019;18(1):35.

16. Wang X, Policarpio L, Prajapati D, Li Z, Zhang H. Developing E. coli-E. coli co-cultures to overcome barriers of heterologous tryptamine biosynthesis. Metab Eng Commun. 2020;10:e00110.

17. Zhang H, Wang X. Modular co-culture engineering, a new approach for metabolic engineering. Metab Eng. 2016;37:114-21.

18. Guo X, Li Z, Wang X, Wang J, Chala J, Lu Y, Zhang H. De novo phenol bioproduction from glucose using biosensor-assisted microbial coculture engineering. Biotechnol Bioeng. 2019;1 16:3349-59.

19. Guo X, Wang X, Chen T, Lu Y, Zhang H. Comparing E. coli mono-cultures and co-cultures for biosynthesis of protocatechuic acid and hydroquinone. Biochem Eng J. 2020;156:107518.

20. Alper H, Stephanopoulos G. Global transcription machinery engineering: a new approach for improving cellular phenotype. Metab Eng. 2007;9:258-67.

21. Wang X, Cabales A, Li Z, Zhang H. Biosensor-assisted high performing cell selection using an E. coli toxin/antitoxin system. Biochem Eng J. 2019;144:110-8
22. Zhang H, Pereira B, Li Z, Stephanopoulos G. Engineering Escherichia coli coculture systems for the production of biochemical products. Proc Natl Acad Sci U S A. 2015:112:8266-71.

23. Kim HG, Doronina NV, Trotsenko YA, Kim SW. Methylophaga aminisulfidivorans sp. nov., a restricted facultatively methylotrophic marine bacterium. Int J Syst Evol Microbiol. 2007;57:2096-101.

24. Clomburg JM, Gonzalez R. Anaerobic fermentation of glycerol: a platform for renewable fuels and chemicals. Trends Biotechnol. 2013;31:20-8.

25. Mattam AJ, Clomburg JM, Gonzalez R, Yazdani SS. Fermentation of glycerol and production of valuable chemical and biofuel molecules. Biotechnol Lett. 2013;35:831-42.

26. Trondle J, Trachtmann N, Sprenger GA, Weuster-Botz D. Fed-batch production of I-tryptophan from glycerol using recombinant Escherichia coli. Biotechnol Bioeng. 2018;1 15:2881-92.

27. Rugbjerg P, Sarup-Lytzen K, Nagy M, Sommer MOA. Synthetic addiction extends the productive life time of engineered Escherichia coli populations. Proc Natl Acad Sci USA. 2018;115:2347-52.

28. Xiao Y, Bowen CH, Liu D, Zhang F. Exploiting nongenetic cell-to-cell variation for enhanced biosynthesis. Nat Chem Biol. 2016;12(5):339-44.

29. Xu X, Li X, Liu Y, Zhu Y, Li J, Du G, et al. Pyruvate-responsive genetic circuits for dynamic control of central metabolism. Nat Chem Biol. 2020;16(11):1261-8.

30. Martínez K, de Anda R, Hernández G, Escalante A, Gosset G, Ramírez OT, et al. Coutilization of glucose and glycerol enhances the production of aromatic compounds in an Escherichia coli strain lacking the phosphoenolpyruvate: carbohydrate phosphotransferase system. Microb Cell Fact. 2008; $7: 1$.

31. Gottlieb K, Albermann C, Sprenger GA. Improvement of L-phenylalanine production from glycerol by recombinant Escherichia coli strains: the role of extra copies of glpK, glpX, and tktA genes. Microb Cell Fact. 2014;13:96.

32. Li G, Young KD. A CAMP-independent carbohydrate-driven mechanism inhibits tnaA expression and TnaA enzyme activity in Escherichia coli. Microbiology. 2014;160:2079-88.

33. Alper H, Moxley J, Nevoigt E, Fink GR, Stephanopoulos G. Engineering yeast transcription machinery for improved ethanol tolerance and production. Science. 2006;314:1565-8.

34. Davis JH, Rubin AJ, Sauer RT. Design, construction and characterization of a set of insulated bacterial promoters. Nucleic Acids Res. 2011;39(3):1131-41

\section{Publisher's Note}

Springer Nature remains neutral with regard to jurisdictional claims in published maps and institutional affiliations.

\footnotetext{
Ready to submit your research? Choose BMC and benefit from:

- fast, convenient online submission

- thorough peer review by experienced researchers in your field

- rapid publication on acceptance

- support for research data, including large and complex data types

- gold Open Access which fosters wider collaboration and increased citations

- maximum visibility for your research: over $100 \mathrm{M}$ website views per year
}

At BMC, research is always in progress.

Learn more biomedcentral.com/submissions 